\title{
A METHODOLOGY TO GENERATE DESIGN ALLOWABLES OF COMPOSITE LAMINATES USING MACHINE LEARNING
}

\author{
C. Furtado ${ }^{1,2(*)}$, L. F. Pereira ${ }^{2,3,4}$, R. P. Tavares ${ }^{5}$, M. Salgado ${ }^{2,3}$, F. Otero ${ }^{6}$, G. Catalanotti ${ }^{7}$, A. \\ Arteiro $^{2,3}$, M. A. Bessa ${ }^{4}$, P. P. Camanho ${ }^{2,3}$ \\ ${ }^{1}$ Dept. of Aeronautics and Astronautics, Massachusetts Institute of Technology, Cambridge, MA, USA \\ ${ }^{2}$ DEMec, Faculdade de Engenharia, Universidade do Porto, Porto, Portugal \\ ${ }^{3}$ INEGI, Porto, Portugal \\ ${ }^{4}$ Dept. of Materials Science and Engineering, Delft University of Technology, Delft, The Netherlands \\ ${ }^{5}$ Dept. of Materials, Textiles and Chemical Engineering, Ghent University, Ghent, Belgium \\ ${ }^{6} \mathrm{CIMNE}$, Barcelona, Spain \\ ${ }^{7}$ ACRG, School of Mechanical and Aerospace Engineering, Queen's University Belfast, Belfast, UK \\ (*)Email: cfurtado@mit.edu
}

The generation of design allowables for composite laminates is of utmost importance for the design and certification of the composite structures used in the aerospace industry. The determination of these design allowables, which account for the variability associated with curing procedures, geometrical features and defects, usually relies on expensive and timeconsuming experimental test campaigns. With the increase of computational power, and the development of high-fidelity numerical models that accurately represent the response of composite materials, alternatives to generate design allowables based on finite element simulations have also been sought out to reduce the certification costs. However, these solutions are still computationally expensive, especially when uncertainty is accounted for. The recent advances on machine learning techniques opens a new window of possibilities for the faster prediction of the structural response of materials, by allowing the definition of surrogate models that continuously and analytically describe the design space [1].

In this work, a feasibility study on the application of machine learning techniques for predicting a design allowable, the notched strength of multidirectional composite laminates, is presented, with the main goal of addressing the challenges of applying machine learning techniques to describe failure of composite laminates and to evaluate the most appropriate algorithms for the determination of composite design allowables.

Building on data generated analytically [2], four machine algorithms are used to predict the notched strength of composite laminates and their statistical distribution, associated to material and geometrical variability. Regarding the representation of the design space, Gaussian Processes models were able to achieve the best performances for very small number of data points, whereas Artificial Neural Networks outperformed all the algorithms for increasing number of data points. The models were also shown to accurately represent the statistical distribution of open-hole strength, thus giving good estimation for the B-value design allowable. This work serves as basis to tackle a more demanding future challenge: the prediction of first-ply failure strength, ultimate strength and failure mode of composite materials based on finite element simulations.

\section{References}

[1] Bessa MA, et al. A framework for data-driven analysis of materials under uncertainty: Countering the curse of dimensionality. Computer Methods in Applied Mechanics and Engineering 2017

[2] Furtado C, et al. Prediction of size effects in open-hole laminates using only the Young's modulus, the strength, and the R-curve of the $0^{\circ}$ ply. Composites Part A: Applied Science and Manufacturing 2017 